\title{
Línea jurisprudencial sobre el reconocimiento de derechos a la comunidad LGTBIQ*
}

\author{
Jurisprudential line on the recognition of rights to the LGTBIQ community \\ Darlis Johana Altamiranda Morales ${ }^{1} \boldsymbol{\otimes}$, Sara Rebeca Mendoza Ríos ${ }^{2} \boldsymbol{\otimes}$, \\ María Fernanda Medina Ruiz ${ }^{3 凶}$, Adiley Carmona Montoya ${ }^{4 凶}$
}

Fecha correspondencia:

Recibido: 30 de junio de 2020.

Revisión: 9 de noviembre de 2020.

Aceptado: 10 de noviembre de 2020

Forma de citar:

Altamiranda, Darlis Johana;

Mendoza, Sara Rebeca; Medina,

María Fernanda; Carmona, Adiley.

"Línea jurisprudencial sobre el

reconocimiento de derechos a la

comunidad LGTBIQ." En: Revista

CES Derecho. Vol. 11, No. 2, junio a

diciembre de 2020, 25-40.

Open access

Términos de uso

Licencia creative commons

Etica de publicaciones

Revisión por pares

Gestión por Open Journal System

DOI: http://dx.doi.org/10.21615/

cesder.11.2.2

ISSN: 2145-7719

Sobre el artículo:

Artículo resultado del trabajo

realizado en el Semillero de

Pensiones de la Facultad de

Derecho de la Universidad CES.

Sobre los autores:

1. Estudiante de derecho.

Universidad CES.

2. Estudiante de derecho.

Universidad CES.

\section{Resumen}

El presente artículo tiene como objetivo realizar el estudio y análisis de las sentencias existentes frente al reconocimiento y reivindicación de derechos a la población LGTBIQ; con el fin de lograr una revisión adecuada frente a los pronunciamientos hechos en el tiempo por la Corte Constitucional. Para ello, se realizó una línea jurisprudencial en la cual se utiliza la técnica propuesta por el académico Diego López en su libro "el derecho de los jueces" y que corresponde al Análisis Dinámico de la Jurisprudencia; es decir, se establece una pregunta o problema jurídico a resolver y se abre un abanico de posibles respuestas a través del análisis de las decisiones judiciales.

Se desarrolló este análisis a través de un enfoque de tipo argumentativo, exploratorio y de tipo documental, pues se basó en la revisión de los conceptos de la Corte Constitucional emitidos en relación con la población LGTBIQ. Esta exploración se realizó como insumo para la realización de un proyecto de investigación relacionado con el reconocimiento de derechos pensionales a la misma comunidad.

Palabras Clave: LGTBIQ, Derechos fundamentales, Corte Constitucional, Línea jurisprudencial, Análisis dinámico.

\section{Abstract}

The objective of this article is to carry out the study and analysis of the existing judgments regarding the recognition and claim of rights to the LGTBIQ population; In order to achieve an adequate review against the pronouncements made over time by the constitutional court, a jurisprudential line was made for this, using the technique proposed by the academic Diego López in his book "the right of judges "And that corresponds to the Dynamic Analysis of Jurisprudence; that is, a legal question or problem to be solved is established and a range of possible answers is opened through the analysis of judicial decisions.

This analysis was developed through an argumentative, exploratory, and documentary type approach, since it was based on the review of the concepts of the Constitutional Court issued in relation to the LGTBIQ population. This exploration was carried out as an input to carry out a research project related to the recognition of pension rights to the same community. 
3. Estudiante de derecho. Universidad CES.

4. Abogada, magíster en Seguridad Social de la Unviersidad CES. Docente de la Facultad de Derecho de la Universidad CES y de la Universidad Católica del Oriente.
Keywords: LGTBIQ, Fundamental rights, Constitutional Court, Jurisprudential line, Dynamic analysis.

\section{Introducción}

En Colombia, a partir de la Constitución Política de 1991 y con la implementación de la figura de la Corte Constitucional se inició un proceso de reconocimiento y reivindicación de derechos que ha llevado a algunos grupos poblacionales comúnmente conocidos como "minorías" a exigir el respeto y la aplicación en condiciones de igualdad de esas garantías constitucionales que en la carta política se encuentran en los primeros artículos.

Entre esos grupos poblacionales que han luchado incansablemente por la reivindicación de sus derechos se encuentra la comunidad LGTBIQ (lesbianas, gays, transexuales, transgénero, bisexuales, intersexuales y queer) que a partir de sentencias de la Corte Constitucional que datan del año 1993 empezaron a solicitar la protección de derechos a la igualdad, la salud, la vida, el libre desarrollo de la personalidad, entre otros (Corte Constitucional, 1993).

Gracias a esa lucha y al nacimiento del movimiento LGTBIQ múltiples entidades, asociaciones y fundaciones, la mayoría sin ánimo de lucro se han dado a la tarea de apoyar a estas personas en sus batallas jurídicas, lo que ha implicado que hoy por hoy se tenga un precedente jurisprudencial muy nutrido en relación con las garantías constitucionales de esta población, llevando cada vez más a la inclusión y el respeto de la diversidad.

Atendiendo a lo anterior, surgió en el semillero de pensiones de la Facultad de Derecho de la Universidad CES la inquietud sobre el reconocimiento de derechos pensionales a la comunidad LGTBIQ, sin embargo, para responder a la pregunta que suscitaba ese tema era necesario primero abarcar un estudio sobre el reconocimiento de derechos de manera general a la comunidad, buscando evidenciar la mayoría de temas tratados en sentencias de la Corte Constitucional y determinar si este reconocimiento se daba en virtud de la condición de población de especial protección o por el hecho de que quien la impulsaba era un ser humano con derechos y deberes. Para llevar a cabo el mencionado análisis de recurrió a la realización de una línea jurisprudencial en la cual se utiliza la técnica propuesta por el académico Diego López Medina en su libro "El derecho de los jueces" y que corresponde al análisis dinámico de la jurisprudencia; es decir, se establece una pregunta o problema jurídico a resolver y se abre un abanico de posibles respuestas a través del análisis de las decisiones judiciales.

Este artículo se realiza bajo un enfoque cualitativo, hermenéutico, documental, exploratorio, toda vez que se partió de la delimitación de un objeto de estudio, se establecieron unas categorías de análisis y a partir de allí se recurrió a la búsqueda de sentencias de la Corte Constitucional con el fin de identificar los pronunciamientos relacionados con ese objeto de estudio.

\section{Línea jurisprudencial de reconocimiento de derechos a la comunidad LGTBIQ}

El procedimiento utilizado para realizar la línea jurisprudencial de reconocimiento de derechos a la comunidad LGTBIQ, consiste en partir de una sentencia que es el punto de apoyo, para el caso en concreto se utilizó la más reciente relacionada con 
población LGTBIQ; posteriormente, se aplicó la ingeniería en reversa, la cual permite identificar las sentencias que harán parte del nicho citacional; y finalmente, realizar un análisis de cada una de ellas y definir aquellas sentencias conocidas como fundadoras, consolidadoras, modificadoras y dominantes, observación que finalmente permitirá encontrar la solución al problema jurídico en alguno de los dos polos de respuesta (López, 2015).

Por otra parte, se considera importante, para el actual panorama de reivindicación de derechos por parte de la comunidad LGTBIQ en el mundo, identificar si en Colombia el reconocimiento de derechos se realiza con argumentos relacionados a la protección de un grupo minoritario y discriminado a través de los años, o si se efectúa con el argumento de reivindicar derechos humanos a cualquier persona independiente de su inclinación sexual o de género.

Para responder lo planteado, se realiza la estructuración de la línea jurisprudencial identificando el punto de apoyo o arquimédico, la sentencia fundadora, y el complemento del nicho citacional. Para lo cual, se analizan las siguientes sentencias:

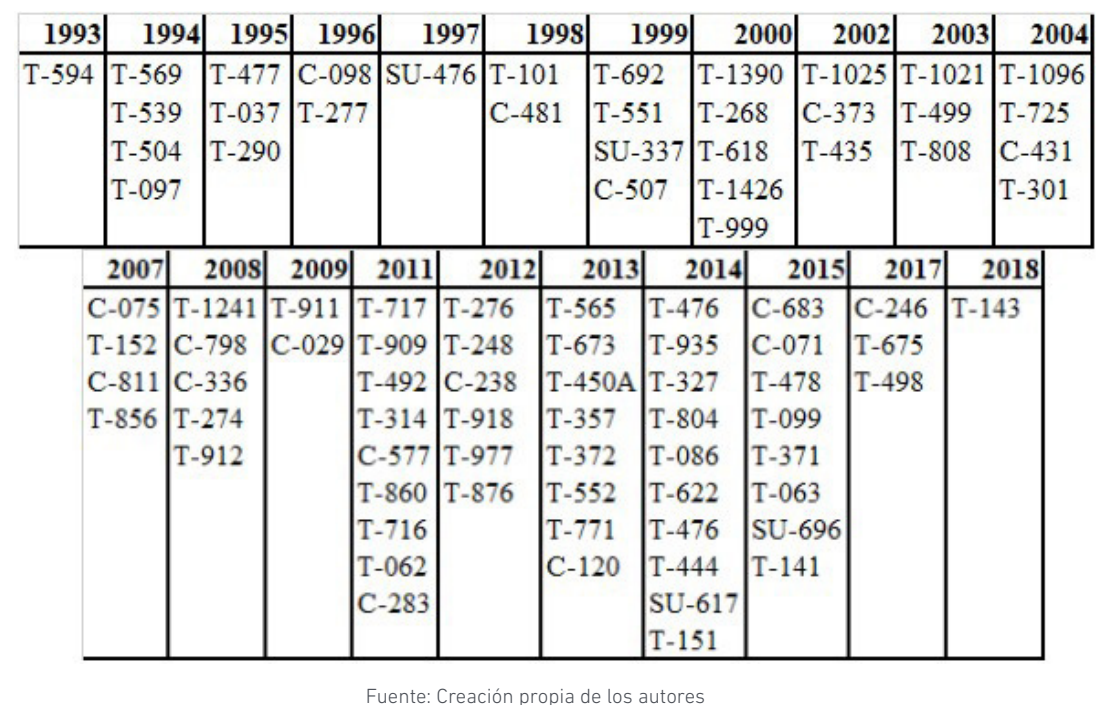

De la ingeniería en reversa visualizada se deriva la siguiente línea jurisprudencial junto con su respectivo nicho citacional.

\begin{tabular}{|c|c|c|c|c|}
\hline & & T-143/2018 & & \\
\hline T-675/2017 & T-099/2015 & T-141/2018 & T-476/2014 & T-804/2014 \\
\hline & & & & \\
\hline T-918/2012 & T-476/2014 & T-476/2014 & T-477/1995 & T-476/2014 \\
\hline T-594/1993 & T-804/2014 & T-804/2014 & C- $075 / 2007$ & T-101/1998 \\
\hline $\mathrm{T}-450 \mathrm{~A} / 2013$ & T-918/2012 & T-086/2014 & & C-075/2007 \\
\hline T-622/2014 & T-062/2011 & T-771/2013 & & \\
\hline & C-577/2011 & T-918/2012 & & \\
\hline & C- $075 / 2007$ & T-876/2012 & & \\
\hline & T-1021/2003 & T-062/2011 & & \\
\hline & \begin{tabular}{|l} 
T-551/1999 \\
\end{tabular} & C-577/2011 & & \\
\hline & C-507/1999 & C- $075 / 2007$ & & \\
\hline & T-565/2013 & T-101/1998 & & \\
\hline & $\mathrm{T}-450 \mathrm{~A} / 2013$ & $\mathrm{~T}-450 \mathrm{~A} / 2013$ & & \\
\hline
\end{tabular}


Como se puede observar dentro de la gráfica, es posible determinar que el punto arquimédico o sentencia de apoyo es la T-143/2018; ésta sentencia trata el caso de un trabajador de una caja de compensación que nació y fue registrado bajo el sexo femenino, pero aduce identificarse con el sexo masculino, y solicita a la entidad empleadora se le cambie el uniforme de trabajo puesto que resalta su cuerpo femenino situación que le resulta incómoda.

Su solicitud fue negada con base en las políticas organizacionales de la entidad y debido a que en su documento de identificación aparece con sexo femenino y así fue adelantado el proceso de selección. El trabajador inicia acción de tutela en contra de la entidad bajo los fundamentos de la violación de sus derechos a la identidad de género, dignidad humana, personalidad jurídica y el libre desarrollo de la personalidad. Si bien, la decisión de la Corte consistió en declarar la carencia actual de objeto, toda vez que para la época en que fue revisada la decisión el empleador del tutelante ya le había hecho entrega de un uniforme correspondiente al sexo masculino, también realizó un amplio análisis de los derechos vulnerados en un caso como el que era objeto de estudio y su relación con la población LGTBIQ.

En este sentido la Corte Constitucional realizó un análisis de la dignidad humana como un principio del estado social de derecho, resaltando que la misma es un pilar fundamental para el correcto goce de los demás derechos fundamentales, y que exige respetar "a cada individuo como es, con sus rasgos característicos y diferencias específicas, en tanto 'esa individualidad es la que distingue cada sujeto de la especie humana" (Corte Constitucional, 2018).

Así mismo, reconoce que existe el derecho al libre desarrollo de la personalidad que establece la posibilidad de que un individuo desarrolle en la sociedad un rol determinado por él mismo y atendiendo a las múltiples opciones que ofrece la vida en los campos privado y público, reconoce que en consecuencia se encuentra el derecho a tener identidad de género que va relacionada con la forma como una persona se desarrolla en el entorno social, adoptando cualidades de un género u otro que puede o no corresponder con el sexo biológico.

Finalmente establece que dentro de la identidad de género se encuentran diferentes variables, una de ellas es reconocida como transgenerismo que tiene que ver con "la no conformidad entre el sexo biológico de la persona y la identidad de género que ha sido tradicionalmente asignada a este" (Corte Constitucional, 2018), además que este grupo se encuentra dentro del reconocido LGTBIQ que ha padecido durante décadas discriminación y exclusión social, y que por ende requieren mayor atención por parte del estado y sus instituciones.

De estos argumentos se desprende que la Corte realiza el reconocimiento del derecho al libre desarrollo de la personalidad, al accionante por pertenecer a la comunidad LGTBIQ, y por ende requerir una mayor protección de sus derechos por parte del Estado.

Posteriormente, se analiza el grupo de sentencias hito, dentro de la cual se encuentra la fundadora de línea, que corresponde a la T-594 de 1993; la cual describe el caso del ciudadano Carlos Montaño, quien interpuso acción de tutela en contra del Notario Tercero del Círculo de Cali, puesto que en ésta entidad se le negó la petición de cambiar su nombre actual por el de Pamela Montaño Díaz con el cual se 
identificaba hacía 13 años; pues implicaba el cambio de un nombre de sexo masculino a otro de sexo femenino.

En principio la Corte realiza un análisis de la individualidad del ser humano, y la necesidad que tienen las personas de ser reconocidas como ente distinto y distinguible, así como el deber de la sociedad y el Estado de respetar este derecho, que encuentra su límite únicamente en los derechos de los demás, el orden público y el bien común.

Así mismo, la Corte manifiesta que el derecho al libre desarrollo de la personalidad: el cual tiene como fundamento la autonomía del hombre como persona, y para ello el Estado reconoce la facultad de toda persona de ser individualmente como quiere. Manifiesta también, que el nombre tiene como finalidad fijar la identidad de las personas, lo cual les permite ser reconocidas en los entornos sociales y ante el Estado; por tal razón, en Colombia existe la posibilidad jurídica de realizar el cambio de nombre a través de una escritura pública que será inscrita en el registro civil de la persona, dicha posibilidad está permitida por una sola vez. Considera la Corte que no existen razones jurídicas para que un funcionario se niegue a cambiar un nombre masculino por uno femenino, pues cada persona desde su individualidad toma la determinación de identificarse como considere que corresponda a su modo de ser, convicción, pensamiento, etc.(Corte Constitucional, 1993).

La Corte realiza la salvedad que en el caso concreto no se está solicitando el cambio de sexo, por lo que este ítem en el registro civil debe permanecer igual, pero si el cambio de nombre, por lo que el notario debe limitarse a dar autenticidad a un documento que se realiza por la voluntad única y expresa del accionante.

Si bien en esta sentencia la Corte Constitucional no tutela el derecho al accionante por pertenecer a la comunidad LGTBIQ, es un primer antecedente para el reconocimiento de derechos a una comunidad que ha sido discriminada a través del tiempo, lo que otorga la posibilidad de invocar este fallo buscando la igualdad en casos similares, por ello se considera la sentencia fundadora de línea.

Así mismo, son estudiadas las sentencias consolidadoras de línea; dentro de las que se pueden observar la T-918 DE 2012, T-099 DE 2015, T-675 DE 2017, T-804 DE 2014, T-086 DE 2014, de acuerdo con el análisis de dichas sentencias es posible encontrar:

\section{Sentencia T-918/2012}

Los hechos relatan la respuesta negativa de la EPS frente a una solicitud de cirugía de "vaginoplastia con intestino y genitoplastia feminizante", para realizar cambio de sexo que fue recomendado por el especialista encargado del cuidado de la accionante; argumentando que no existía ningún riesgo para su vida; dicha justificación es empleada por la EPS en solicitudes realizadas en años posteriores. Además, se encuentra que la accionante fue diagnosticada con trastorno de identidad de género "Síndrome de Harry Benjamín; con base en éste, el especialista en urología adscrito a la E.P.S. ordenó la realización de los procedimientos de vaginoplastia, orquidectomía simple y penectomía total, pero hasta el momento en que se interpuso la acción de tutela no había respuesta alguna por parte de la entidad.

La Corte realiza el análisis partiendo de una reiteración de jurisprudencia acerca del derecho a la identidad sexual; inicialmente define la dignidad y esgrime que es 
una base suprema, que consiste en que cada persona debe ser tratada acorde a su naturaleza humana, que en definitiva es la que permite establecer la definición de identidad sexual:

En el derecho a la identidad la persona es un ser autónomo, con autoridad propia, orientado a fines específicos, que ejerce un claro dominio de su libertad y en consecuencia ninguna decisión tomada sin su consentimiento se torna válida. Tal autonomía, implica a la persona como dueña de su propio ser. La persona por su misma plenitud es dueña de sí, es el sujeto autónomo y libre. En otros términos, el distintivo de ser persona y el fundamento de la dignidad de la persona es el dominio de lo que quiere ser. (Corte Constitucional, 2012)

Es así como la corte establece a manera de conclusión que cobra vital importancia la salvaguarda de los derechos fundamentales al libre desarrollo de la personalidad, a la autodeterminación y la dignidad humana, y aclara de manera contundente que el Estado no puede imponer barrera alguna para que el individuo decida su desarrollo vital, su modo de ser y su condición sexual.

\section{Sentencia T-099/2015}

Se describe el caso de una persona que al momento de su nacimiento fue identificada como hombre, pero con el pasar del tiempo manifestó sentirse mujer y empezó a tener atracción hacia los hombres; debido a circunstancias personales acaba en el mundo de la prostitución en el cual desafortunadamente contrae el virus del papiloma humano; igualmente es víctima de constantes amenazas a su vida y es forzada a desplazarse de su domicilio.

Para el caso concreto, la corte realiza un ejercicio de derecho comparado acerca de los derechos que son internacionalmente reconocidos a las personas trans y cita importantes referencias como los principios de Yogyakarta o el pronunciamiento expreso de la Corte Interamericana de Derechos Humanos y la Corte Europea de Derechos Humanos.

Al finalizar el análisis la corte establece que la dignidad humana es la autonomía de los sujetos para diseñar un plan de vida y determinarse según sus características; además, implica que los ciudadanos puedan vivir libres de cualquier tipo de ofensa. La dignidad tiene un nexo inescindible con los derechos al libre desarrollo de la personalidad, a la autonomía y a la identidad personal, potestades cuyo ejercicio transciende el plano individual, por tal razón sus manifestaciones públicas deben ser objeto de protección sin perjuicio de los límites que imponen los derechos de los demás y el mantenimiento del orden. Generalmente, en estos escenarios de expresión de los derechos se presentan situaciones que pueden ser violatorias del derecho a la igualdad debido a eventuales reacciones discriminatorias. (Corte Constitucional 2015)

\section{Sentencia T-675 DE 2017}

Sentencia que se refiere al caso de Juan Andrés, quien considera que no existe correspondencia entre el sexo asignado por la conformación y características genitales con las que nació, con su real y verdadera identidad sexual, la cual se basa en una orientación netamente femenina que ha venido acentuándose y marcándose cada vez más por el transcurso de los años. Por tal razón, a sus 17 años opta por reconocerse en su ámbito familiar, escolar y social como María Alejandra; en consecuencia, la Madre del menor solicita a la notaria hacer el correspondiente cambio en 
el componente sexo y nombre en el documento de identidad; no obstante la entidad desconoce solicitud y recomienda que se instaure una acción de tutela; la cual fue denegada argumentando que según el decreto 1227/2015 para realizar los cambios correspondientes en el documento de identidad es necesario haber cumplido la mayoría de edad (Corte Constitucional, 2017).

La Corte inicia el estudio desde los dogmas de la constitución donde la misma se atreve a afirmar que el libre desarrollo a la personalidad, derecho en cuestión; configura una verdadera garantía a las identidades de género diversas, puesto que, las personas trans tienen la potestad de escoger libremente su plan de vida, tal y como lo pueden hacer el resto de individuos en Colombia, mismo que no corresponde única y exclusivamente al ámbito interno y personal, sino que puede manifestarse públicamente al contar con plena protección constitucional. De lo contrario, evitar la trascendencia social de una persona trans implicaría un entendimiento abiertamente contrario a los postulados constitucionales.

Es así como la Corte afirma que los derechos al libre desarrollo de la personalidad y a la dignidad humana son de carácter supremo y las infracciones a los mismos configuran una falta a los principios y fines del Estado.

Ordena se realice el cambio en el componente sexo, sentencia que además es el antecedente más importante para la regulación para el cambio del componente sexo en menores de edad, permitiendo que se expida la instrucción administrativa 12 de 2018 de la Superintendencia de Notariado y Registro. (Supernotariado, 2018)

\section{Sentencia T-804 DE 2014}

Los hechos relevantes descritos en la sentencia se refieren al caso de un joven transgénero que intenta matricularse en una institución educativa para cursar el grado 11, sin embargo, el docente a cargo de su admisión debido a su aspecto; es decir, por la forma en la que vestía, se negó a otorgar el cupo en la institución; razón por la cual se exige el respeto del derecho a la educación, sin discriminación por razones de identidad de género.

La Corte precisa la existencia de una reiteración de jurisprudencia, en torno a los derechos fundamentales como la dignidad humana, igualdad, así como el libre desarrollo de la personalidad. Fundamentando el alcance, extensión e importancia del derecho a la dignidad humana así:

La importancia de la dignidad humana, considerada por esta Corporación como "derecho fundante del Estado" y "presupuesto esencial de la consagración y efectividad del entero sistema de derechos y garantías contemplado en la Constitución", en la medida que implica reconocer la persona como un fin en sí mismo y exige un trato especial por el individuo. (Corte Constitucional, 2014).

Así mismo, se reconoce a través de la jurisprudencia constitucional, que el núcleo esencial de la dignidad humana "supone que la persona sea tratada de acuerdo con su naturaleza humana y el Estado, dentro de sus fines esenciales, debe preservar la libertad, la autonomía, la integridad física y moral, la exclusión de tratos degradantes, la intimidad personal y familiar". En esa medida, el respecto de la dignidad humana implica aceptar a cada individuo como es, con sus rasgos característicos y diferencias específicas, en tanto "esa individualidad es la que distingue cada sujeto de la especie humana" (Corte Constitucional, 2014). 
Es con sustento en lo anterior que el ordenamiento constitucional desarrolla otras garantías fundamentales como los derechos a la igualdad y al libre desarrollo de la personalidad. En virtud del artículo 13 de la Constitución, todas las personas nacen libres e iguales ante la ley, por lo que deben recibir el mismo trato y protección sin ninguna discriminación por razones de sexo, raza, origen nacional, etc.

\section{Sentencia T-086 DE 2014}

La sentencia gira en torno a Mario, individuo que nació de sexo masculino y cambió en una primera instancia su nombre a uno femenino en la notaría sin ningún inconveniente; sin embargo, al sentirse identificado con su cuerpo masculino solicita nuevamente cambio de nombre; momento para el cual la Registraduría y la Superintendencia de Notariado y Registro, siguiendo los parámetros de ley dan respuesta negativa a la solicitud del accionante.

La Corte establece en primer plano la definición del significado y connotación que tiene el nombre; el cual encuentra por finalidad contribuir a fijar la identidad de la persona desde su nacimiento, frente a la sociedad en general y al Estado. Posteriormente, es reiterada la prohibición a múltiples cambios de nombre. Por supuesto, el objeto de análisis desde el derecho a la identidad de género y dignidad humana de las personas homosexuales establece y reitera la jurisprudencia expresando lo siguiente:

La protección de los derechos fundamentales al libre desarrollo de la personalidad, a la dignidad humana y a la identidad, ha reconocido la facultad que tiene todo ser humano de desarrollarse y comportarse acorde con sus gustos y preferencias. También ha indicado que, el nombre, además de ser un atributo de la personalidad, es un elemento definitorio de la persona que la distingue de las demás. Este generalmente coincide con la identidad sexual, pero cuando no lo hace, está dentro del marco de autonomía de las personas cambiarlo. En ese orden de ideas, el Estado debe respetar y garantizar la libre decisión del individuo respecto de su identidad, para evitar menoscabar su dignidad (Corte Constitucional, 2014).

Sin embargo, la Corporación ha reconocido que la restricción prevista en el artículo 94 del decreto 1260 es razonable y proporcional, en la medida en que lo que pretende es garantizar un mínimo de seguridad jurídica en las relaciones sociales y permitir al Estado administrar adecuadamente las bases de datos públicas y evitar incongruencias que afecten derechos fundamentales. A pesar de ello, la jurisprudencia de la Corte ha inaplicado la norma y, por ende, ha permitido por segunda vez el cambio de nombre, cuando se trata de una persona que ha tenido un proceso en su desarrollo de identidad sexual y de género, y su nombre no coincide con la apariencia física asumida (Corte Constitucional, 2014).

De las sentencias consolidadoras de línea es posible inferir que los derechos reconocidos a los miembros de la comunidad LGBTIQ van de la mano a los utópicos derechos que, aunque son principios y fines constitucionales, y deben ser objeto de protección por parte del estado colombiano, a la fecha no hay una verdadera tutela para los mismos; no es suficiente entonces solo reconocer estos derechos, sino que hay que buscar la verdadera protección y como se hace efectiva la misma; ya que de acuerdo a lo expresado en distintas ocasiones por la Corte, los derechos que se reconocen de forma masiva y que son reiterados a la comunidad son derechos que en general todas las personas por el simple hecho de haber nacido tienen; lo que 
conlleva a solo garantizar la igualdad a todos los seres humanos del Estado; pero ¿cuáles derechos se les reconoce a los miembros de la comunidad LGBTIQ?

Ahora bien, dentro del rastreo jurisprudencial también pueden encontrarse las sentencias confirmadoras de línea; dentro de las cuales se obtuvieron: T-1021 DE 2003, C-075 DE 2007, T-551 DE 1999, T-771 DE 2013, T-876 DE 2012, T-476 DE 2014, T-062 DE 2011, T-622 DE 2014 y T-101 DE 1998.

\section{Sentencia T-1021 DE 2003}

La sentencia tiene como origen la acción de tutela que interpone la madre de un menor con diagnóstico médico de hermafroditismo, lo que conlleva a realizar operaciones para el cambio de sexo. La entidad promotora de salud solo exige a la accionante que realice el pago de una cuota de recuperación, por lo que la acción de tutela va encaminada a solicitar la protección del derecho por la falta de dinero de la familia, buscando que no se cobre la cuota de recuperación; por lo cual, la Corte inicia su estudio, adentrándose además en el estudio del problema jurídico que se venía presentando alrededor del consentimiento informado cuando se trata de niños intersexuales. Así mismo, aprovecha para reiterar la jurisprudencia frente al alcance del derecho al libre desarrollo de la personalidad y dignidad humana de las personas de la comunidad LGTBIQ.

La Corte expone lo que significa el entendimiento de la sexualidad humana; la cual no se reduce a la asignación de la calidad de hombre o mujer, derivada de la evaluación de la apariencia física, sino que también incluye otras variables, tales como el moldeamiento comportamental del género, la preferencia sexual, etc., que en el actual modelo constitucional adquieren reconocimiento y son protegidas al ser expresiones del ejercicio de los derechos fundamentales a la autonomía individual y al libre desarrollo de la personalidad (Corte Constitucional, 2003)

Finalmente, la Corte expresa específicamente lo que es el derecho al libre desarrollo de la personalidad y su significado para los individuos que al nacer tienen la condición de intersexuales, de la siguiente manera:

Los procedimientos de remodelación genital y reasignación de sexo comportan profundas implicaciones con el ejercicio del derecho fundamental al libre desarrollo de la personalidad, ya que de este derecho se derivan un cúmulo de atributos y garantías a favor del individuo, entre ellas el derecho a la identidad personal y a la definición sexual, que se ven afectados en grado sumo con las decisiones médicas que se tomen con el objeto de superar un estado intersexual. Además, los procedimientos quirúrgicos, terapéuticos y hormonales a los que se viene haciendo referencia son altamente invasivos, requieren, en el común de los casos, de varias intervenciones y, como se dijo, carecen de la posibilidad de reversarse.

Bajo estos supuestos, caracterizados por la intensa afectación de derechos fundamentales constitucionales y la alta complejidad de la intervención médica, resulta perentorio que el paciente autorice los procedimientos sanitarios en el marco de un consentimiento libre, informado y cualificado, lo que exige un alto grado de autonomía personal y capacidad suficiente para tomar decisiones trascendentales sobre la propia salud y opción de vida. (Corte Constitucional, 2003).

Es de reconocer que, en los casos similares al expuesto, debe hacerse énfasis en que el derecho les pertenece a los intersexuales; ya que no se trata de la salud de todos, si no del bienestar y la salud de la minoría en referencia. 


\section{Sentencia C-075 DE 2007}

En la sentencia se ejerce la acción pública de inconstitucionalidad, donde se demandan parcialmente los artículos $1^{\circ}$ y $2^{\circ}$ de la Ley 54 de 1990 "por la cual se definen las uniones maritales de hecho y el régimen patrimonial entre compañeros permanentes", modificada parcialmente por la Ley 979 de 2005. Los actores consideran que cuando el legislador definió la figura de "compañeros permanentes" como aquella conformada exclusivamente por dos personas heterosexuales omitió la posibilidad de que una pareja del mismo sexo conformase un proyecto de vida en común, lo que conlleva a generar un impacto negativo sobre las personas homosexuales, también señalan que ese impacto se concreta en que las uniones homosexuales no tienen acceso a todos aquellos derechos (patrimoniales y extrapatrimoniales) que se derivan del reconocimiento a la unión consagrada en la Ley 54 de 1990.

La Corte para esgrimir dicho dilema; realiza un análisis desde el ámbito internacional teniendo en cuenta los conceptos del Comité de Derechos Humanos de Naciones Unidas, órgano responsable de la interpretación del Pacto Internacional de Derechos Civiles y Políticos para determinar que:

Específicamente se han producido distintos pronunciamientos orientados a identificar los casos en los que la diferencia de tratamiento entre parejas heterosexuales y homosexuales puede considerarse una forma de discriminación debido a la orientación sexual" (Corte Constitucional, 2007).

No toda distinción equivale a la discriminación prohibida por el Pacto Internacional de Derechos Civiles y Políticos, en la medida en que se basa en criterios razonables y objetivos; por lo tanto, las diferencias objetivas que existen entre los dos tipos de pareja, y los factores específicos considerados por el legislador del año 1990 para establecer este régimen de protección, fundado en la necesidad de proteger a la familia; no son impedimento para advertirse que las parejas homosexuales presentan requerimientos análogos de protección y que no existen razones objetivas que justifiquen un tratamiento diferenciado.

De esta manera; la Corte manifiesta que la norma no amerita una diferenciación con las parejas homosexuales debido a que podría, entonces, discriminar a la minoría. Finalmente concluye qué frente a las parejas del mismo sexo, solo cuando sea necesario, se debe hacer diferenciación, con el fin de no vulnerar su derecho a la dignidad humana, al libre desarrollo de la personalidad e identidad de género.

\section{Sentencia T-551 DE 1999}

En esta oportunidad se examina el caso de una niña de dos años que presenta una forma de ambigüedad genital, a saber, un pseudohermafroditismo femenino por hiperplasia suprarrenal congénita. Su padre, interpone acción de tutela solicitando que se le tutelen los derechos a la salud, a la seguridad social, a la igualdad y al libre desarrollo de la personalidad de la menor; ordenando al ISS que lleve a cabo una cirugía para remodelar los genitales de la menor, y que además le suministre todos los medicamentos y terapias "que sean necesarios para enfrentar esa dolencia" (Corte Constitucional, 1999).

La Corte Constitucional luego de conocer el proceso médico de la menor y verificar que se le realizó la cirugía y los tratamientos, realiza un análisis implícito acerca del consentimiento sustituto paterno para llevar a cabo la cirugía, poniendo de presente que se deben tener en cuenta tres criterios: "(i) la necesidad y urgencia del 
tratamiento, (ii) su impacto y riesgos, y (iii) la edad y madurez del menor" (Corte Constitucional, 1999)

La Corte concluye que la remodelación de genitales no es una práctica rutinaria y común, sino que es una práctica invasiva, riesgosa y discutida; por lo tanto, no debe mediar consentimiento del padre, puesto que se está protegiendo el interés superior del menor. De este modo, decide tutelar los derechos a la intimidad, a la identidad sexual y al libre desarrollo de la personalidad de la menor.

\section{Sentencia T-771 DE 2013}

En esta sentencia se revisa el caso de una mujer transgénero, que interpone acción de tutela en contra de Comfenalco Antioquia EPS, para que se le amparen los derechos fundamentales al libre desarrollo de la personalidad, a la salud y el derecho a la vida digna; los cuales fueron vulnerados por la EPS al negar los tratamientos necesarios para el cambio de sexo aduciendo que no contaban con recursos económicos y científicos suficientes para tratar la disforia de género. En Bogotá, Compensar EPS le niega nuevamente los tratamientos puesto que no son cubiertos por el POS y además tienen fines estéticos.

La Corte establece que el POS no puede negar la realización del tratamiento argumentado que es un cambio estético; puesto que las transformaciones físicas hacen parte de la construcción identitaria de, en este caso, las mujeres trans. Por ello, dicha transformación física no se realiza con fines estéticos, sino que hace parte de la identidad de género de la persona.

Además, establece que el aumento mamario en este caso no solo tiene un carácter funcional, sino que es la forma de llevar a la práctica el derecho que asiste a la accionante de construir su propio concepto de feminidad. De acuerdo con lo anterior, la Corte concede el amparo a los derechos fundamentales a la salud, la vida digna, a la identidad sexual y al libre desarrollo de la personalidad a la actora y ordena que se realicen los procedimientos que se requieren en el proceso de reafirmación sexual quirúrgica (Corte Constitucional, 2013).

\section{Sentencia T-876 DE 2012}

Se analiza la tutela interpuesta para que sean amparados los derechos a la salud y a la vida digna de un hombre, quien en 1985 nació identificado como del sexo femenino pero que desde sus primeros años de vida y hasta ser adulto se ha desarrollado y es conocido por todos como del sexo masculino. El accionante se encuentra afiliado al régimen subsidiado de la EPS COMPARTA, donde asistió a constantes terapias y tratamientos psicológicos, producto de los cuales fue diagnosticado con transexualismo; derivado de ello se debe someter a una cirugía de cambio de sexo, la cual fue negada en primera oportunidad por la EPS por no estar dentro del POS.

La Corte en sentencia T-307 de abril 19 de 2006 afirmó que "la salud no equivale únicamente a un estado de bienestar físico o funcional. Incluye también el bienestar psíquico, emocional y social de las personas. Todos estos aspectos contribuyen a configurar una vida de calidad e inciden fuertemente en el desarrollo integral del ser humano" (Corte Constitucional 2006).

Luego de que la Corte estudiara la jurisprudencia, establece que el derecho a la salud es fundamental; motivo por el cual la EPS, al abstenerse de suministrar o 
autorizar un tratamiento excluido del POS amenaza el bienestar de la persona, al negar un procedimiento médico que requiere un usuario, para superar situaciones que afecten su bienestar físico, mental y social. De acuerdo con la situación fáctica del caso, la Corte observa que la EPS COMPARTA y la Secretaría de Salud de Cundinamarca sí vulneraron los derechos invocados por el actor, por no autorizar la cirugía de cambio de sexo, razón por la que se tutelan los derechos fundamentales a la salud y la vida digna (Corte Constitucional, 2012).

\section{Sentencia T-476 DE 2014}

En el texto referido se hace alusión a una mujer transgénero que interpone acción de tutela en contra de la Subdirección de Asuntos LGBT de la Secretaría de Integración Social de la Alcaldía Mayor de Bogotá y la Dirección Nacional de Reclutamiento del Ejército Nacional, al considerar vulnerados sus derechos fundamentales al trabajo, al libre desarrollo de la personalidad, al mínimo vital y a la vida en condiciones dignas; puesto que la Secretaría en cuestión negó la posibilidad de continuar en un proceso de contratación por no poseer el requisito de libreta militar, el cual se dice es indispensable para la contratación con entidades públicas.

La Corte establece que en los procesos de selección y contratación que se adelanten entre entidades públicas y particulares, no se podrá exigir libreta militar a las personas transgeneristas. Esto en atención a las especiales condiciones con las que cuentan las personas de la comunidad LGTBI; y es por esto por lo que ni los particulares ni el Estado pueden obstaculizar la formación de la identidad de género de las personas (Corte Constitucional, 2014).

De tal manera, se les permite a las personas que construyan sus propios proyectos de vida acorde a su identidad de género, con la limitación de los derechos de las otras personas y el orden jurídico.

Frente a lo expuesto, la Corte decide amparar los derechos fundamentales al trabajo, al libre desarrollo de la personalidad, a la identidad de género, al mínimo vital y a la vida en condiciones dignas de la accionante.

\section{Sentencia T-062 DE 2011}

En la sentencia se hace revisión de acción de tutela interpuesta por un interno del Establecimiento Penitenciario de Mediana Seguridad de Yopal destinada a lograr su protección al libre desarrollo de la personalidad; a través de órdenes a sus funcionarios, para que se abstengan de continuar con los malos tratos, y le restituyan y permitan usar los objetos decomisados como lo fueron los aretes, el kit de maquillaje y las moñas para recoger el cabello.

La Corte establece que las conductas realizadas por el interno al interior de la cárcel como lo es usar maquillaje, vestirse con prendas de mujer y tener el pelo largo, refuerzan la adopción de su identidad sexual; lo cual es manifestación de la autonomía personal, y la privación injustificada de los mismos conlleva a la vulneración de sus derechos a la dignidad humana, el libre desarrollo de la personalidad y la igualdad (Corte Constitucional, 2011).

Las decisiones tomadas al interior del establecimiento penitenciario solo fueron basadas en una aparente violación de reglamentos, no teniendo en cuentas las normas 
que regulan este caso, fundamentándose dichas decisiones en que la diversidad social es una condición de anormalidad y que atenta con la moral social.

La Corte Constitucional decide amparar los derechos pedidos por el tutelante.

\section{Sentencia T-622 DE 2014}

En este caso la madre de un menor de 11 años presenta acción de tutela para que se le protejan a su hijo los derechos fundamentales a la dignidad humana, identidad sexual, seguridad social y a la salud. Expresando que su hijo presenta ambigüedad sexual, diagnosticada como hermafroditismo masculino, por lo que requiere que el niño tenga intervención psicológica y médica, más aún considerando los tratamientos discriminatorios que ha presentado en su entorno social y académico. El menor expresa querer pertenecer al sexo masculino por lo que se requiere de intervención quirúrgica la cual no ha sido autorizada por la EPS SURA. Igualmente, es importante tener en cuenta que no se ha cumplido con los requisitos necesarios para obtener un consentimiento informado.

Por otro lado, para dar respuesta al problema jurídico, la Sala observa que no existía orden del médico tratante que requiriera la cirugía de reasignación de sexo, y esta situación se debe a que no se ha realizado un debido tratamiento del caso del menor. De manera que, la Corte encuentra una vulneración de los derechos fundamentales del niño por la ausencia de un diagnóstico certero, luego de casi 10 meses de consultas y remisiones, que demuestran una falta de atención médica eficiente y oportuna acorde con su condición.

La Corte decide amparar los derechos del menor ordenando a la EPS SURA continuar con las evaluaciones pertinentes por parte de los especialistas y determinar si es procedente la cirugía, caso en el cual se debe realizar parte de la EPS. Además, ordena al ICBF que realice acompañamiento al menor y a su familia y a la Registraduría Nacional por su parte que cambie el sexo en la tarjeta de identidad del menor por el que coincida con la realidad y evitar así tratos discriminatorios (Corte Constitucional, 2014).

\section{Sentencia T-101 DE 1998}

Dos jóvenes eran estudiantes del Instituto Ginebra -La Salle- (Institución oficial). De esta institución se retiran debido a situaciones económicas. Tiempo después deciden volver a estudiar allí; por lo tanto, reclaman la reserva de los cupos. El rector y el consejo directivo de dicha institución niegan la petición, aduciendo que además de ser gays, violaron las normas del manual de convivencia que establece que la solicitud de reserva del cupo debe hacerse de manera escrita ante el Consejo Directivo de la Institución. Los actores, quienes son menores de edad presentan acción de tutela con el fin de que se proteja el derecho a la educación y a libre desarrollo de la personalidad.

La Corte encuentra que, acorde con los expedientes el rector de la institución utiliza términos discriminatorios en distintas oportunidades para referirse a los menores de edad, lo que denota que la decisión de este y del consejo directivo estuvo influenciada por la identidad sexual de los actores. Además, se evidencia que en el reglamento de la Institución se establece que se rige por la religión católica; lo que es una situación claramente violatoria de la Constitución, pues al ser un colegio oficial no se debe imponer ninguna religión a los estudiantes, dicha situación probablemente 
también haya influenciado a las directivas de la Institución para adoptar la decisión de denegar los cupos a los exestudiantes (Corte Constitucional, 1998).

La Corte además logró poner de presente que no se viola ninguna norma del manual de convivencia por parte de los menores puesto que el manual solo exige que la solicitud de reserva se realice de manera escrita para los estudiantes activos, pero nada establece para los exestudiantes que desean ingresar nuevamente a la Institución. Por lo tanto, se considera que se violenta el derecho a la igualdad, a la educación y al libre desarrollo de la personalidad a los menores (Corte Constitucional, 1998).

Finalmente, se obtiene la sentencia dominante de línea, reconocida por ser aquella que se encuentra citada en la mayoría de las sentencias de la línea, no solo cuando se trata de personas intersexuales, sino también cuando la Corte debe referirse a derechos de personas de la comunidad LGTBI, relacionados con derechos como el nombre, la personalidad jurídica y la identidad sexual.

\section{Sentencia T-450 A DE 2013}

En esta sentencia se discute el caso de un niño intersexual, donde en el certificado de nacido vivo no se especifica el sexo del bebé quedando registrado en ese campo como "NN" y por esta razón los funcionarios de la Registraduría del Estado Civil se negaron a registrarlo; obstaculizando de este modo el acceso del menor a los servicios de salud y en general a la garantía de sus derechos como ciudadano colombiano.

La Corte inicia su análisis examinando el derecho a la personalidad jurídica; manifestando que este no solo se materializa en la consideración del individuo como titular de derechos y deberes, sino que "por el simple hecho de existir se le garanticen los atributos que conforman la esencia de la personalidad" (Corte Constitucional 2013).

Reitera entonces la Corte que uno de esos atributos de la personalidad que son inalienables es el nombre, característica que además es considerado un derecho fundamental, este permite identificar a las personas en la sociedad y otorgarle un vínculo familiar. Así mismo, considera que el derecho a la personalidad jurídica y a la identidad tiene íntima relación con la dignidad humana.

Frente al caso concreto, manifiesta que la no identificación del sexo en una persona no puede ser un motivo para negarle derechos de vital importancia, entre ellos, la personalidad jurídica, la identidad y el nombre; por lo que un funcionario público no puede escudarse en normas jurídicas para negar estos derechos a personas intersexuales en quienes resuelta complejo definir un sexo u otro desde el momento del nacimiento (Corte Constitucional, 2013).

La Corte considera que existe un vacío legal en la materia, pues no hay reglas claras que deban seguir los funcionarios públicos cuando se encuentren frente a una situación como la del caso concreto, y por lo tanto exhorta al Congreso de la República para que se legisle frente al tema. 


\section{Conclusiones}

En un número mayor de las sentencias analizadas, la Corte Constitucional reivindica el derecho de las personas accionantes atendiendo a criterios de universalidad, es decir, por ser derechos humanos, no por la distinción de género o sexo.

La línea jurisprudencial tiene como base los derechos al nombre, la identidad de género, la personalidad jurídica, libre desarrollo de la personalidad, dignidad humana, estableciendo el precedente constitucional de protección especial a la población LGTBIQ en todas aquellas garantías constitucionales que les han sido amenazadas o vulneradas por sus diferentes decisiones de vida e identidad de género.

A la fecha la Corte Constitucional en materia pensional sólo se ha pronunciado frente al reconocimiento de pensión de sobrevivientes a parejas del mismo sexo, toda vez que, han sido los únicos problemas jurídicos ventilados en esta corporación, existiendo aún la posibilidad de encontrar personas transgénero que libran batallas jurídicas buscando el reconocimiento de pensiones bajo criterios de igualdad, o la unificación de historias laborales cotizadas bajo identidades de género diferentes.

\section{Referencias}

López, Diego. (2006). El derecho de los jueces, Bogotá, Legis.

Corte Constitucional Colombiana, Sentencia T-594 del 15 de diciembre de 1993, disponible en: https://www.corteconstitucional.gov.co/relatoria/1993/t-594-93.htm

Corte Constitucional Colombiana, Sentencia T-143 del 23 de abril de 2018, disponible en: https://www.corteconstitucional.gov.co/relatoria/2018/T-143-18.htm

Corte Constitucional Colombiana, Sentencia T-918 del 08 de noviembre de 2012, disponible en: https://www.corteconstitucional.gov.co/relatoria/2012/T-918-12.htm

Corte Constitucional Colombiana, Sentencia T-099 del 10 de marzo de 2015, disponible en: https://www.corteconstitucional.gov.co/relatoria/2015/t-099-15.htm\#: : :tex$\mathrm{t}=\mathrm{T} \%$ 2D099\%2D15\%20Corte\%20Constitucional\%20de\%20Colombia\&text=La\%20 identidad\%20de\%20g\%C3\%A9nero\%20y,se\%20apropia\%20de\%20su\%20sexualidad.

Corte Constitucional Colombiana, Sentencia T-675 del 15 de noviembre de 2017, disponible en: https://www.corteconstitucional.gov.co/relatoria/2017/T-675-17.htm

Corte Constitucional Colombiana, Sentencia T-804 del 04 de noviembre de 2014, disponible en: https://www.corteconstitucional.gov.co/RELATORIA/2014/T-804-14. $\underline{\mathrm{htm}}$

Corte Constitucional Colombiana, Sentencia T-086 del 17 de febrero de 2014, disponible en: https://www.corteconstitucional.gov.co/relatoria/2014/T-086-14.htm

Corte Constitucional Colombiana, Sentencia T-1021 del 30 de octubre de 2003, disponible en: https://www.corteconstitucional.gov.co/relatoria/2003/t-1021-03. htm

Corte Constitucional Colombiana, Sentencia C-075 del 07 de febrero de 2007, disponible en: https://www.corteconstitucional.gov.co/relatoria/2007/c-075-07.htm

Corte Constitucional Colombiana, Sentencia T-551 del 02 de agosto de 1999, disponible en: https://www.corteconstitucional.gov.co/relatoria/1999/t-551-99.htm

Corte Constitucional Colombiana, Sentencia T-771 del 07 de noviembre de 2013, disponible en: https://www.corteconstitucional.gov.co/relatoria/2013/T-771-13.htm

Corte Constitucional Colombiana, Sentencia T-876 del 29 de octubre de 2012, disponible en: https://www.corteconstitucional.gov.co/relatoria/2012/t-876-12.htm

Corte Constitucional Colombiana, Sentencia T-307 del 19 de abril de 2006, disponible en: https://www.corteconstitucional.gov.co/relatoria/2006/t-307-06.htm 
Corte Constitucional Colombiana, Sentencia T-476 del 09 de julio de 2014, disponible en: https://www.corteconstitucional.gov.co/relatoria/2014/T-476-14.htm

Corte Constitucional Colombiana, Sentencia T-062 del 04 de febrero de 2011, disponible en: https://www.corteconstitucional.gov.co/relatoria/2011/t-062-11.htm

Corte Constitucional Colombiana, Sentencia T-622 del 28 de agosto de 2014, disponible en: https://www.corteconstitucional.gov.co/relatoria/2014/t-622-14.htm

Corte Constitucional Colombiana, Sentencia T-101 del 24 de marzo de 1998, disponible en: https://www.corteconstitucional.gov.co/relatoria/1998/t-101-98.htm

Corte Constitucional Colombiana, Sentencia T-450A del 16 de julio de 2013, disponible en: https://www.corteconstitucional.gov.co/relatoria/2013/t-450a-13.htm 